\title{
Fermi Surface and Antiferromagnetism in Europium Metal
}

\author{
Andersen, O. Krogh; Loucks, T. L.
}

Published in:

PHYSICAL REVIEW

Link to article, DOI:

10.1103/PhysRev.167.551

Publication date:

1968

Document Version

Publisher's PDF, also known as Version of record

Link back to DTU Orbit

Citation (APA):

Andersen, O. K., \& Loucks, T. L. (1968). Fermi Surface and Antiferromagnetism in Europium Metal. PHYSICAL REVIEW, 167(2), 551-556. https://doi.org/10.1103/PhysRev.167.551

\section{General rights}

Copyright and moral rights for the publications made accessible in the public portal are retained by the authors and/or other copyright owners and it is a condition of accessing publications that users recognise and abide by the legal requirements associated with these rights.

- Users may download and print one copy of any publication from the public portal for the purpose of private study or research.

- You may not further distribute the material or use it for any profit-making activity or commercial gain

- You may freely distribute the URL identifying the publication in the public portal

If you believe that this document breaches copyright please contact us providing details, and we will remove access to the work immediately and investigate your claim. 


\title{
Fermi Surface and Antiferromagnetism in Europium Metal*
}

\author{
O. KROGH ANDERSEN \\ Laboratory for Electrophysics, The Technical University, Lyngby, Denmark \\ AND \\ T. L. Loucks $\dagger$ \\ Institute for Atomic Research and Department of Physics, Iowa State University, Ames, Iowa
}

(Received 12 October 1967)

\begin{abstract}
We have calculated the Fermi surface of europium in order to find those features which determine the wave vector of the helical moment arrangement below the Néel point. We find that there are two pieces of Fermi surface: an electron surface at the symmetry point $H$, which has the shape of a rounded-off cube, and a hole surface at the point $P$, which is also a rounded-off cube (half the size of the one at $H$ ) but with ellipsoids tetrahedrally positioned on four of the corners. We propose that the wave vector of the helical moment arrangement is fixed by the separation between opposing faces of the nearly cubical part of the hole surface at $P$, and we also discuss the effects of the electron surface at $H$. Since it is likely that barium and europium have similar Fermi surfaces, we have presented several extremal areas and the corresponding de Haas-van Alphen frequencies in the hope that experimental results may soon become available.
\end{abstract}

\section{INTRODUCTION}

$\mathbf{O}^{\mathrm{N}}$ $\mathrm{NE}$ of the two divalent rare-earth metals is europium $\left(4 f^{7} 6 s^{2}, \mathrm{bcc}\right)$, and it is isoelectronic with the alkali-earth metal barium $\left(6 s^{2}, \mathrm{bcc}\right) .^{1}$ These two metals are known to have similar electronic structures because they exhibit nearly identical optical spectra..$^{2-4}$ From this it can be concluded that the $4 f$ electrons have energies outside the range of the occupied conduction band.

There is very little known experimentally about these metals, especially those properties which relate directly to the Fermi surface. This situation undoubtedly results from the difficulties encountered in preparing and handling high-purity single crystals. Measurements have been made, however, which give information on the magnetic properties of europium. Curry et al. ${ }^{5}$ reported electrical-resistivity measurements which exhibited a sharp bump around $90^{\circ} \mathrm{K}$, and they concluded that the metal was antiferromagnetic (AF) below this temperature. \{Note added in proof. Recent heat capacity experiments indicate a sharp transition in the neighborhood of $88^{\circ} \mathrm{K}$ [B. C. Gerstein et al., J. Chem. Phys. 47, $5194(1967)]$.$\} Nereson et al .^{6-8}$ confirmed this with a series of neutron-diffraction experiments (they found a

* Work was performed in part in the Ames Laboratory of the U.S. Atomic Energy Commission. Contribution No. 2198.

+ Alfred P. Sloan Research Fellow.

${ }^{1}$ The other divalent rare earth is ytterbium $\left(4 f^{14} 6 s^{2}, \mathrm{fcc}\right)$, and it is isoelectronic with $\mathrm{Ca}\left(4 s^{2}, \mathrm{fcc}\right)$ and $\mathrm{Sr}\left(5 s^{2}, \mathrm{fcc}\right)$.

2 W. E. Mueller, Phys. Letters 17, 82 (1965).

${ }^{3}$ C. C. Scheuler, Optical Properties and Electronic Structure of Metals and Alloys, edited by F. Abeles (North-Holland Publishing Co., Amsterdam, 1966), p. 221.

${ }_{4}^{4}$ W. E. Mueller, Solid State Commun. 4, 581 (1966).

${ }^{5}$ M. A. Curry, S. Legvold, and F. H. Spedding, Phys. Rev. 117, $953(1960)$.

${ }^{6}$ G. P. Arnold, C. E. Olsen, and N. G. Nereson, J. Appl. Phys. Suppl. 33, 1135 (1962).

7 C. E. Olsen, N. G. Nereson, and G. P. Arnold, J. Appl. Phys. Suppl. 35, 1031 (1964).

${ }^{8}$ N. G. Nereson, C. E. Olsen, and G. P. Arnold, Phys. Rev. 135, A176 (1964).
Néel temperature $T_{N}$ of $91^{\circ} \mathrm{K}$ ). They proposed a helical model in which the magnetic moments lie parallel to a cube face and the rotation axis is directed perpendicular to the moments or along the [100] direction. The pitch of the helix was observed to change very slightly with temperature; the period is $3.5 a$ at $T_{N}$ and increases to only $3.6 a$ at $T / T_{N}=0.05$ ( $a$ is the lattice constant).

It was this occurrence of a noncommensurate periodic moment arrangement (PMA) in europium which originally attracted our attention. In two other cases, this phenomenon is known to be related to certain features of the Fermi surface. In chromium, for instance, it was proposed by Lomer ${ }^{9}$ that the period of the spin-density wave is determined by the separation between two approximately parallel electron and hole sheets of the Fermi surface (the well-known electron jack and hole octahedron). There is considerable evidence to support this hypothesis, both theoretical ${ }^{10-12}$ and experimental. ${ }^{13-15}$ The other case is the AF hexagonal close-packed (hcp) rare earths: $\mathrm{Tb}, \mathrm{Dy}, \mathrm{Ho}, \mathrm{Er}$, and Tm. It was proposed by Williams et al. ${ }^{16}$ that approximately parallel electron and hole sheets of the Fermi surface are influential in determining the wave vector of the PMA and also accounted for the unusual positronannihilation results in these metals. This view has been supported by further calculations, ${ }^{17}$ and evidence of

${ }^{9}$ W. M. Lomer, Proc. Phys. Soc. (London) 80, 489 (1962). ${ }^{10}$ P. A. Fedders and P. C. Martin, Phys. Rev. 143, 245 (1966). ${ }^{11} \mathrm{~S}$. Asano and J. Yamashita, Institute for Solid State Physics, Technical Report Series A, No. 262, 1967 (unpublished).

${ }_{12}$ L. M. Falicov and M. J. Zuckermann, Phys. Rev. 160, 372 (1967).

${ }^{13}$ S. Komura, Japan Atomic Energy Research Institute Research Report No. 1137, 1967 (unpublished); S. Komera, Y.

Hamaguchi, and N. Kunitomi, J. Phys. Soc. Japan 23, 171 (1967).

${ }^{14}$ W. C. Koehler, R. M. Moon, A. L. Trego, and A. R. Mackintosh, Phys. Rev. 151, 405 (1966)

${ }^{15} \mathrm{~A}$. R. Mackintosh (to be published).

${ }^{16}$ R. W. Williams, T. L. Loucks, and A. R. Mackintosh, Phys.

Rev. Letters 16, 168 (1966).

${ }^{17}$ S. C. Keeton and T. L. Loucks (to be published). 
this Fermi-surface feature has been found in a variety of experimental results. ${ }^{18-21}$

The theoretical justification for a relationship between the wave vector of a PMA and the "nesting"22 of portions of the Fermi surface has been reviewed by Herring, ${ }^{23}$ and a particularly relevant paper on the subject is given by Roth et al. ${ }^{24}$ We will briefly outline the concepts involved. In a rare-earth metal such as europium there is a magnetic moment associated with the relatively well-localized $4 f$ electrons on each of the ions. These moments are coupled by a RudermanKittel-Yosida indirect exchange interaction involving polarization of the conduction electrons. A consideration of the energy of this system reveals that if the effective susceptibility of the conduction electrons $\chi(\mathbf{q})$ has its maximum at some finite $q=Q$, then the ground state will be an array in which the spins of the magnetic ions have different orientations, i.e., a PMA. Ignoring anisotropy and nonlinear effects, the minimum energy will occur for a helical array with wave vector equal to Q. ${ }^{23}$ The expression for the effective susceptibility of the conduction electrons is proportional to the following summation ${ }^{23}$ :

$$
\begin{aligned}
\chi(\mathbf{q}) \sim \sum_{\mathbf{k}} \eta(\mathbf{k})[1-\eta(\mathbf{k}+\mathbf{q})] \\
\times \frac{(\mathbf{k}+\mathbf{q}|U-A| \mathbf{k})(\mathbf{k}|U| \mathbf{k}+\mathbf{q})}{E(\mathbf{k}+\mathbf{q})-E(\mathbf{k})} .
\end{aligned}
$$

For the present purposes, it is not important to define all the symbols in this expression; we want merely to draw attention to the energy denominator [the Dirac functions $\eta$ simply limit the one-electron levels $E(k)$ to be occupied and $E(k+q)$ to be empty]. If there are approximately parallel portions of the Fermi surface separated in reciprocal space by the distance $Q$, then $\chi(\mathbf{q})$ will have a maximum near $\mathbf{q}=\mathrm{Q}$. We have seen above that this leads to a PMA with wave vector $Q$.

This leads us to suspect that the PMA in europium is probably associated with nesting portions of the Fermi surface. Curiosity on this point was our original motivation for performing the electronic-structure calculations reported in this paper. For completeness, we should point out that prior to the present work, the energy bands and density states had been calculated by

${ }^{18} \mathrm{R}$. W. Williams and A. R. Mackintosh (to be published).

${ }^{19} \mathrm{H}$. Bjerrum M $\phi$ ller and J. C. Gylden Houmann, Phys. Rev. Letters 16, 737 (1966).

${ }^{20} \mathrm{H}$. Bjerrum M $\varnothing l$ lier, J. C. Gylden Houmann, and A. R. Mackintosh, Phys. Rev. Letters 19, 312 (1967).

${ }^{21}$ S. K. Sinha, T. Brun, L. D. Muhlestein, and J. Sakurai (to be published).

${ }_{22}$ "Nesting" is a descriptive term for the situation in which two approximately parallel sheets of the Fermi surface can be made to coincide by displacing either of them an appropriate distance in k space.

${ }^{23} \mathrm{C}$. Herring, Magnetism, edited by G. T. Rado and H. Suhl (Academic Press Inc., New York, 1966), Vol. IV.

2 L. M. Roth, H. J. Zeiger, and T. A. Kaplan, Phys. Rev. 149, 519 (1966).
Freeman and Dimmock. ${ }^{25}$ However, they did not determine the Fermi surface but rather drew attention to the fact that, as in the case of chromium, the Fermi energy falls near a minimum in the density-of-states curve. Prior to our work, no attempt had been made to show a relationship between the Fermi surface and the occurrence of a noncommensurate PMA in europium.

\section{RESULTS}

Europium has atomic number $Z=63$, and relativistic effects can be important. Therefore, we used the relativistic augmented-plane-wave (APW) method ${ }^{26,27}$ for our calculations. In constructing the muffin-tin potential, we used the atomic charge densities of Waber et $a l .^{28}$ for configuration $4 f^{7} 6 s^{2}$. And although there is mounting evidence that Slater free-electron exchange $\left(\rho^{1 / 3}\right)$ probably should be screened a little (by a factor somewhat less than unity of metals) we used the full value. Instead of tabulating the potential, we have shown the logarithmic derivatives (actually $c f / g$, the relativistic analog) in Fig. 1.. ${ }^{29}$ These were evaluated at a muffin-tin sphere radius of $\exp (1.3)=3.67$ a.u., ${ }^{30}$ and the calculations were based on a lattice constant

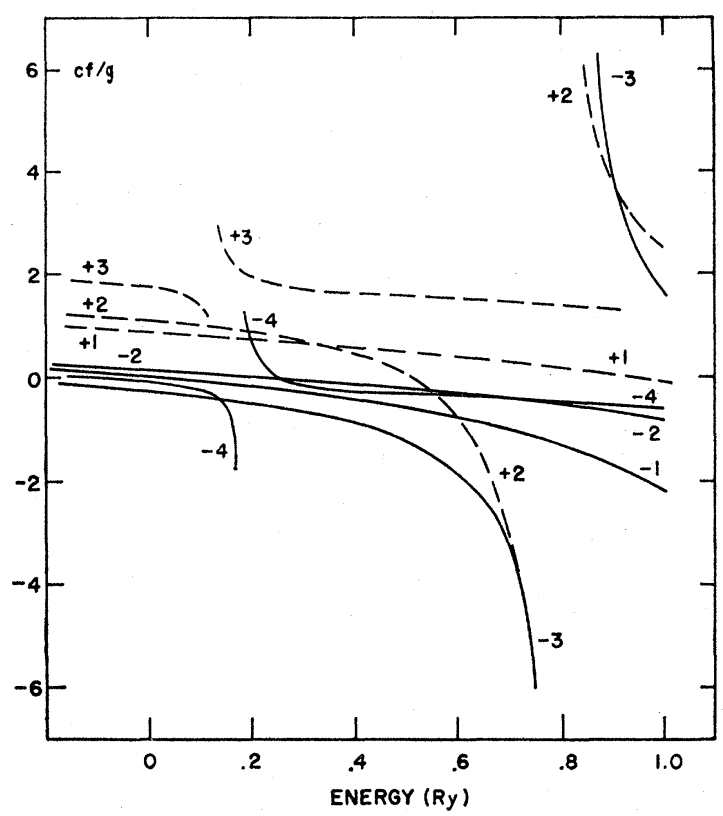

FIG. 1. cf/g values (see Ref. 27) for europium.

${ }^{25}$ A. J. Freeman and J. O. Dimmock, Bull. Am. Phys. Soc. 11, 216 (1966); also (private communications).

${ }^{26}$ T. L. Loucks, Phys. Rev. 139, A1333 (1965).

${ }^{27}$ T. L. Loucks, Augmented Plane Wave Method (W. A. Benjamin, Inc., New York, 1967).

${ }^{28}$ D. Liberman, J. T. Waber, and Don T. Cromer, Phys. Rev. 137, A27 (1965).

${ }_{29}^{29}$ It can be seen from Fig. 1 that the $f$ band $(\kappa=3$ and -4$)$ should fall in the vicinity of $E$ equal to $0.18 \mathrm{Ry}$. Since exchange and correlation effects for the $4 f$ electrons have not been properly treated in this calculation, the position of this band is probably not significant. We have therefore not shown the corresponding energy levels in Fig. 3.

${ }^{30}$ Atomic units (a.u.) are specified by $e^{2}=2, m=\frac{1}{2}$, and $\hbar=1$. 


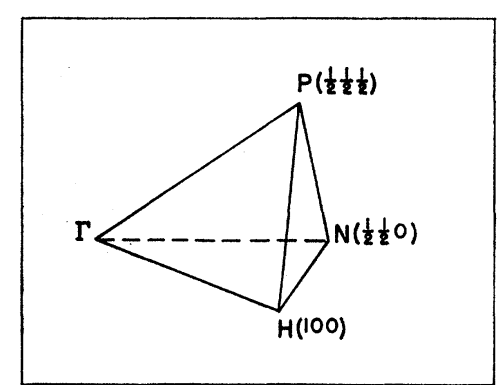

FIG. 2. 1/48th zone of the bcc Brillouin zone.

of 8.60 a.u. ${ }^{31}$ We used 22 reciprocal-lattice vectors because only one Brillouin zone is occupied, and convergence is not a problem. The basis set includes the origin $(0,0,0)$, all those vectors of the form $( \pm \lambda$, $\pm \lambda, 0),( \pm 2 \lambda, 0,0)$, and three additional ones: $(-2 \lambda$, $-2 \lambda, 0),(-2 \lambda, 0,-2 \lambda)$, and $(2 \lambda,-2 \lambda,-2 \lambda)$. Here, $\lambda=2 \pi / a$, the distance between the two symmetry points $\Gamma$ and $H$ in the $1 / 48$ th zone shown in Fig. 2.

The relativistic energy bands for europium are shown in Fig. 3. The Fermi energy (0.287 Ry) was determined by the requirement that the electrons at $H$ and the holes at $P$ occupy equal volumes. The volumes of these portions of the Fermi surface are shown for various energies in Fig. 4. From the slopes of these curves we found the density of states at the Fermi energy to be

$$
N\left(E_{F}\right)=12.0 \text { particles/atom Ry, }
$$

which yields an electronic specific-heat coefficient of

$$
\gamma=2.08 \mathrm{~mJ} / \mathrm{mole}^{\circ} \mathrm{K}^{2} \text {. }
$$

Two different coordinate systems were used to calculate cross sections of the Fermi surface. For the holes at $P$, cylindrical coordinates were used; the $z$ axis is along the edge $P H$ (origin at $P$ ), and the azimuthal angle $\varphi$ is zero in the $N P H$ plane $\left(\varphi=60^{\circ}\right.$ in the $\Gamma P H$ plane). Cross sections of the hole Fermi surface centered at $P$ are shown in Fig. 5 for various values of $\varphi$. For the electrons at $H$, spherical coordinates were used. The $z$ axis is along the edge $H \Gamma$ (origin at $H$ ), and

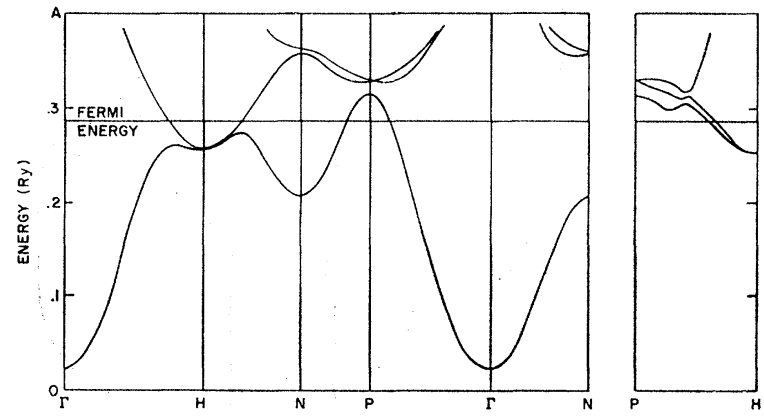

FIG. 3. Relativistic energy bands for europium.

${ }^{31}$ R. W. G. Wyckoff, Crystal Structures (Interscience Publishers, Inc., New York, 1963), Vol. I.

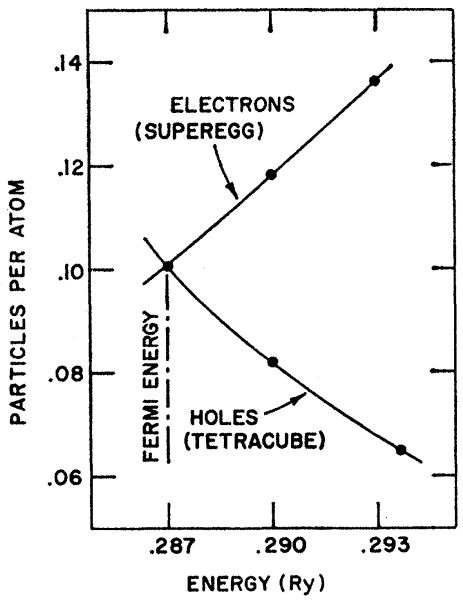

Fig. 4. Volume contained by constant-energy surfaces (given in particles per atom).

the azimuthal angle $\varphi$ is zero in the $H \Gamma N$ plane $\left(\varphi=45^{\circ}\right.$ in the $H \Gamma P$ plane). Cross sections of the electron surface centered at $H$ are shown in Fig. 6 for various values of $\varphi$.

The shapes of the two pieces of Fermi surface can be seen in Figs. 7 and 8. An artist's sketch of the two figures is shown in Fig. 9. The holes at $P$ are shaped like a rounded-off cube with ellipsoids tetrahedrally positioned on four of the corners. We choose to call this the tetracube for ease of reference. The electrons at $H$ are shaped like a rounded-off cube which we call

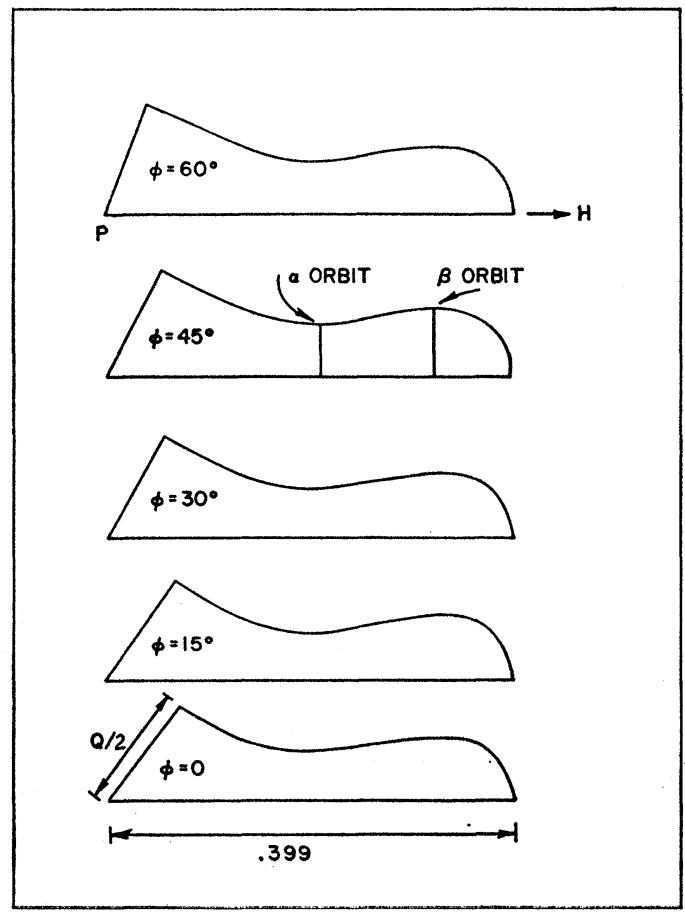

FIG. 5. Cross sections of the hole portion of the Fermi surface (tetracube). 


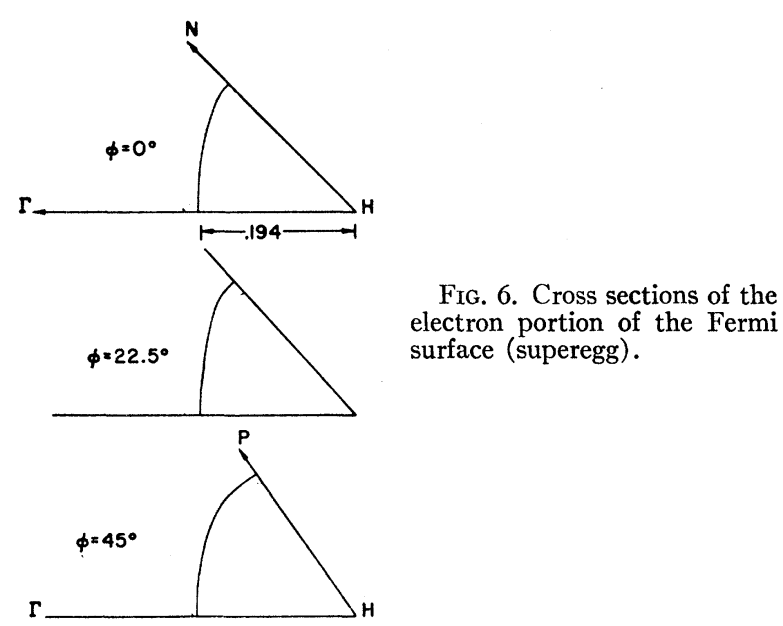

the superegg. ${ }^{32}$ For completeness, we should mention that a slight change in the potential or the Fermi energy can result in the appearance of tiny pieces of Fermi surface inside the tetracube near where the ellipsoids join on to the rounded-off cube. This feature is associated with the minimum in the first band, which occurs about one-third of the distance from $P$ to $H$ (Fig. 3).

We have determined some extremal areas on the tetracube and the superegg. These might be of interest if de Haas-van Alphen data become available for

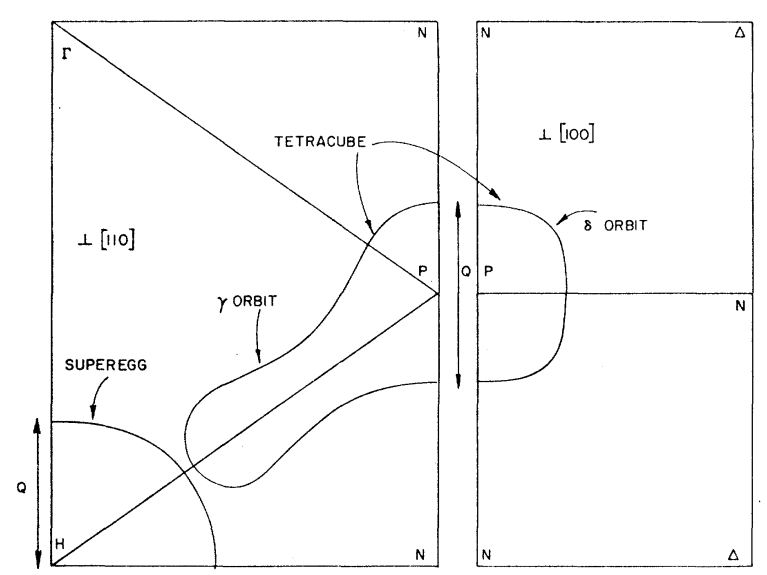

FIG. 7. Cross sections of the tetracube and the superegg.

32 We have chosen the name "superegg" because of the similarity between our rounded-off cube and the "super-egg" designed by Piet Hien, the Danish writer and inventor. In many design applications he has used simple and pleasing shapes which mediate between the round and the rectangular; in two dimensions the curves are called super-ellipses, and in three dimensions he has chosen to call them super-eggs. One family of these surfaces can be described by the equation $x^{n}+y^{n}+z^{n}=$ const. If $n$ is infinite, this reduces to a cube, and if $n=2$, it is the equation for a sphere. For intermediate values of $n$, the equation describes a family of surfaces ranging between these two. We found that $n=13 / 4$ gives a good fit to our Fermi surface except near the corners, where the spin-orbit splitting with the tetracube is important. Literature on the super-egg can be obtained from The Super-Egg World Center, Skjern, Denmark.

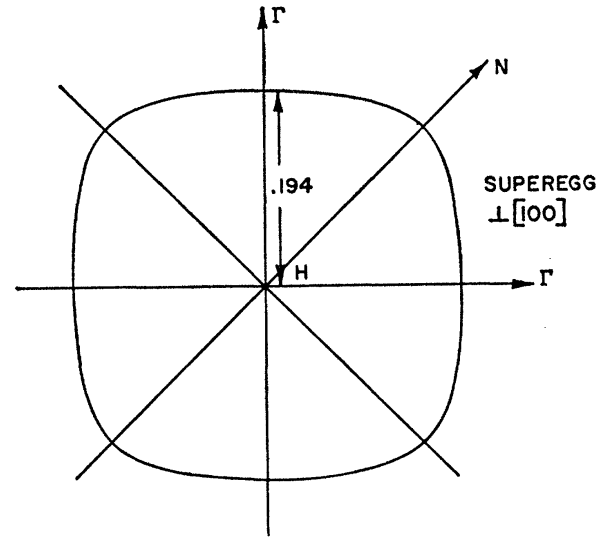

FIG. 8. Cross section of the superegg.

barium before reliable Fermi-surface calculations for this metal have been completed. The results are reported (Table I) as de Haas-van Alphen frequencies (gauss) using the relationship

$$
\begin{gathered}
f \text { (gauss })=\left(374.1 \times 10^{6}\right) \times[\text { extremal area (a.u.) }] . \\
\text { DISCUSSION }
\end{gathered}
$$

The energy bands in Fig. 3 closely resemble those for tungsten ${ }^{33}$ over the same energy range. The most noticeable difference is at the symmetry point $N$, where apparently (we have not actually determined the symmetry of the various levels) the sensitive $p$-like level $N_{1}{ }^{\prime}$ is much lower for europium. The spin-orbit splitting

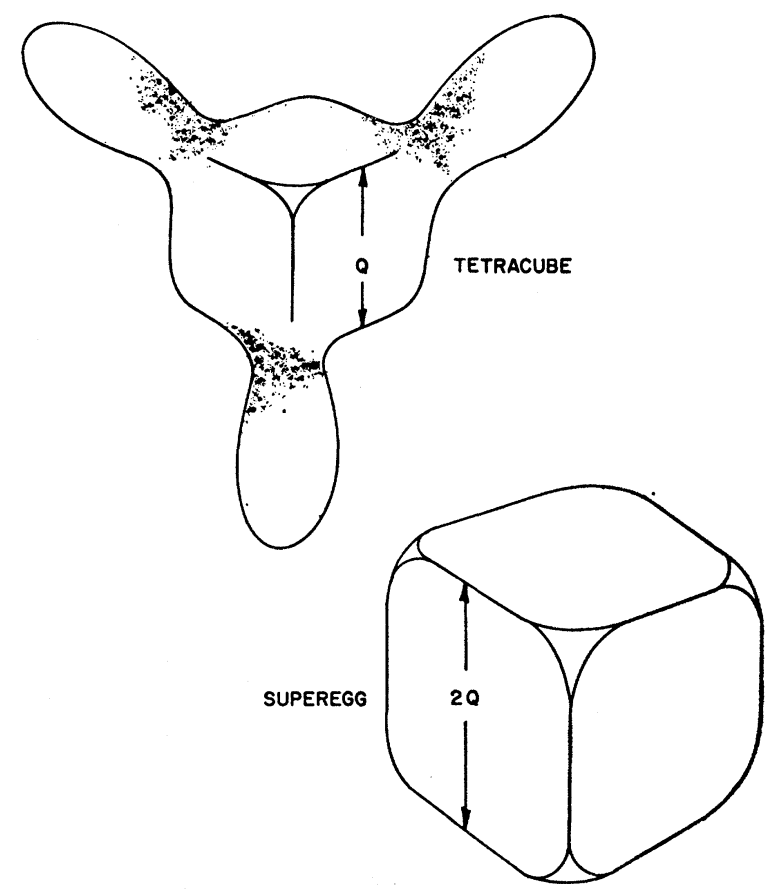

FIg. 9. Europium Fermi surface.

${ }^{33}$ L. F. Mattheiss, Phys. Rev. 139, A1893 (1965). 
of degenerate levels at $P$ and along $P H$ is also worthy of attention. The corresponding splittings in tungsten would be even larger if properly calculated, but the relativistic calculations for this metal ${ }^{34}$ do not include these particular bands. It is apparent that the sizes of both pieces of Fermi surface, the tetracube and the superegg described in the previous section, are dependent on the exact position of the second level at $\mathrm{N}$ and the spin-orbit splitting at $P$ and along $P H$. Since these might depend critically on the potential, we should keep in mind that the one we have used is not necessarily self-consistent and was constructed using an ad hoc procedure.

The first thing we should probably note is the nonfree electron nature of the Fermi surface. The freeelectron result gives multiply connected holes centered at $H$ and electrons at $N$, nothing at all like the results we present. But we should not have expected otherwise. In the atomic state, if one more electron is added to europium (and not into the $4 f$ shell), it would go into the $5 d$ level. Hence, in the band picture we expect the $d$ levels to begin near the Fermi energy corresponding to two electrons per atom. This is seen to be the case in our bands (Fig. 3), and it accounts for the departure from the free-electron result. In this respect europium (and barium) are really transition metals.

Because of the similarity between our europium bands and those of tungsten we can gain some information from the density-of-states curve for the latter. Using the results of Mattheiss ${ }^{33}$ we see that for two electrons per atom the Fermi energy falls near a minimum in the density of states (actually at a somewhat lower energy than the minimum and on a fairly steep part of the curve). This means that calculation of the density of states at the Fermi energy is very sensitive to changes in the potential. Our value [Eq. (2)] gives an electronic specific-heat coefficient [Eq. (3)] which is about one-third as large as the recent experimental value $\left(5.8 \mathrm{~mJ} / \mathrm{mole}{ }^{\circ} \mathrm{K}^{2}\right) .{ }^{35}$ For other transition metals this ratio is usually not so small (more like one-half or greater). There are too many variables (electronphonon enhancement, purity of the sample, steepness

TABLE I. Extremal areas and de Haas-van Alphen frequencies.

\begin{tabular}{llccc}
\hline \hline & Orbit & $\begin{array}{c}\text { Field } \\
\text { direction }\end{array}$ & $\begin{array}{c}\text { Extremal } \\
\text { area } \\
(\text { a.u. })\end{array}$ & $\begin{array}{c}\text { dHvA } \\
\text { frequency } \\
\left(\times 10^{\circ} \mathrm{G}\right)\end{array}$ \\
\hline Tetracube & $\alpha$ (Fig. 5) & {$[111]$} & 0.0079 & 2.9 \\
& $\boldsymbol{\beta}$ (Fig. 5) & {$[111]$} & 0.0125 & 4.7 \\
& $\gamma$ (Fig. 7) & {$[110]$} & 0.109 & 41 \\
Superegg & $\delta$ (Fig. 7) & {$[100]$} & 0.0496 & 19 \\
& $\epsilon$ (Fig. 7) & {$[110]$} & 0.140 & 52 \\
& $\epsilon$ (Fig. 8) & {$[100]$} & 0.131 & 49 \\
\hline \hline
\end{tabular}

${ }^{34}$ T. L. Loucks, Phys. Rev. 143, 506 (1966)

${ }^{35}$ O. N. Lounasmaa, Phys. Rev. 143, $399(1966) ; 133$, A502 (1964).

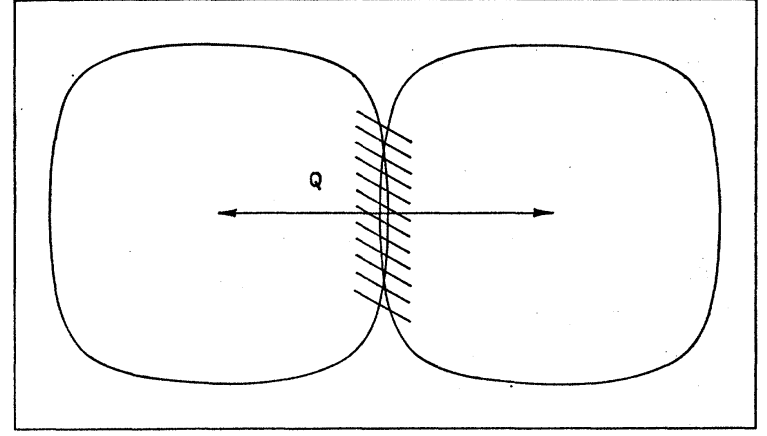

FIG. 10. Hypotheitcal nearly square Fermi surface showing one edge nesting into the opposite one.

of the density-of-states curve, etc.) to attempt to rationalize this further at the present time.

Let us now turn to a consideration of the PMA in europium. In the introduction it was pointed out that nesting portions of the Fermi surface determine the wave vector $\mathbf{Q}$ of the PMA. In europium the $\mathbf{Q}$ is perpendicular to a cube face (along [001]) and has a magnitude (at $T_{N}$ ) equal to $4 \pi / 7 a .^{8}$ With reference to the Brillouin zone (Fig. 2) the wave vector is along the edge $\Gamma H$ (or equivalently $N P$ and $N \Delta$, where $\Delta$ is the midpoint of $\Gamma H)$ and has a magnitude equal to $(2 / 7) \Gamma H$ or $(4 / 7) N P$. In both chromium and in the AF hcp rare earths, the wave vector of the spin density wave (SDW) can be associated with approximately parallel electron and hole portions of the respective Fermi surfaces. It is clear that this cannot be the case in europium, because the superegg (at $H$ ) and the tetracube (at $P$ ) are separated along the [111] direction, instead of along [001] as required by the experimental results. However, it must be remembered that pieces of Fermi surface can sometimes nest into themselves. This is shown in Fig. 10 for a two-dimensional nearly square Fermi surface. In the case of a nearly cubical Fermi surface it is possible for one face to nest into the opposite one.

Apparently this occurs in europium. We recall that the tetracube is essentially a rounded-off cube with ellipsoids tetrahedrally positioned on four of the corners. The important point is that the base of this object is nearly cubical, and opposing faces can nest into one another. According to our calculations the distance between the faces of the tetracube is 0.238 a.u.,$^{30}$ and the $Q$ of the PMA is ${ }^{8} 0.209$ a.u. at $T_{N}$. This agreement suggests very strongly that the $Q$ is fixed by this nesting feature of the tetracube.

There is another interesting feature of the europium Fermi surface. The electron surface at $H$, the superegg, is itself a rounded-off cube and the parallel faces are separated by almost exactly twice the wave vector of the PMA. Specifically, one-half of this separation is found to be 0.194 a.u., compared to the experimental wave vector 0.209 a.u. Therefore, second-order contributions to the susceptibility [Eq. (1) with the energy denominator replaced by $E(k+2 q)-E(k)]$ will assist 
in maintaining a maximum at $q=Q$. [First-order contributions from the superegg will produce a second maximum in $\chi(q)$ at $q=2 Q$.] Since the second-order effects become increasingly important below the Néel point, it is possible that the superegg contribution will help to stabilize the $Q$ of the PMA as a function of temperature (unless, of course, the peak at $2 Q$ should become dominant at some low temperature). Experimentally, the period of the PMA is quite insensitive to temperature. It ranges from $3.5 a$ at $T_{N}$ to $3.6 a$ near $T=0$. We feel that this behavior might be associated with the second-order effects from the nesting of opposing faces of the superegg. The first-order effects of the superegg, i.e., the occurrence of a second maximum in $\chi(q)$ near $q=2 Q$, have not yet been fully examined.

Since there are no other data available relating directly to the Fermi surface, not much more can be said at this time. We can only draw attention to the fact that barium and europium should have similar
Fermi surfaces and encourage further experimental work on both metals. If, for instance, barium also has the nearly cubical pieces of Fermi surface discussed above for europium, then these should be reflected as Kohn-type anomalies in the phonon spectra. There are several ways in which the various flat faces can be nested into one another.

\section{ACKNOWLEDGMENTS}

One of us (TLL) wants to thank the Laboratory for Electrophysics at The Technical University, Lyngby, Denmark for making possible a recent visit. Most of the work reported in this paper was completed there. The friendly and constructive attitude of everyone at the Laboratory provided a very enjoyable and stimulating environment for this activity. It was through the special efforts of Professor A. R. Mackintosh that the visit was a complete success, and both authors are extremely grateful to him for his continued encouragement and advice.

\title{
Mössbauer-Effect Study of Electronic Relaxation in the Paramagnetic Compound $\mathrm{NH}_{4}(\mathrm{Fe}, \mathrm{Al})\left(\mathrm{SO}_{4}\right)_{2} \cdot 12 \mathrm{H}_{2} \mathrm{O} *$
}

\author{
L. E. Campbell $\dagger$ and S. DeBenedetti \\ Carnegie Institute of Technology, Pittsburgh, Pennsylvania
}

(Received 19 October 1967)

\begin{abstract}
The Mössbauer spectra of paramagnetic ferric compounds exhibit magnetic hyperfine splittings when the electronic-relaxation rates of the ferric ions are made comparable to the precession frequency of the nucleus in the hyperfine field. This was observed in ferric ammonium alum diluted in aluminum ammonium alum, $\mathrm{NH}_{4}(\mathrm{Fe}, \mathrm{Al})\left(\mathrm{SO}_{4}\right)_{2} \cdot 12 \mathrm{H}_{2} \mathrm{O}$, as a function of iron concentration, temperature, deuteration, and external magnetic field. The effect was also observed in the corresponding potassium alums. The crystalline-field levels of the ferric ion consist of three Kramers doublets, and each doublet produces its own hyperfine pattern. In the absence of an external magnetic field, the spectrum due to the $S_{z}= \pm \frac{5}{2}$ doublet was com pletely resolved, that due to the \pm 0 doublet partially resolved, and that due to the $\pm \frac{1}{2}$ doublet not resolved at all. Relaxation-time estimates are made ranging from $\approx 10^{-7}$ to $\approx 10^{-10} \mathrm{sec}$ depending on the concentration, the temperature, and the particular Kramers doublet under consideration. The magnetic field produced at the nucleus by the $\pm \frac{5}{2}$ doublet was found to be $-572 \pm 7 \mathrm{kG}$. The isomer shift is $0.53 \pm 0.1 \mathrm{~mm} / \mathrm{sec}$, and the quadrupole-interaction energy, $\frac{1}{4} e^{2} q Q$, is equal to $0.055 \pm 0.025 \mathrm{~mm} / \mathrm{sec}$.
\end{abstract}

\section{INTRODUCTION}

A RELATIVELY new phenomenon in Mössbauer research with $\mathrm{Fe}^{57}$ has been the observation of magnetic hfs in the spectra of paramagnetic absorbers. ${ }^{1-8}$

${ }^{*}$ Work supported by the U.S. Office of Naval Research and the National Science Foundation.

$\dagger$ Present address: Physics Division, Argonne National Laboratory, Argonne, Illinois.

1 G. K. Wertheim and J. P. Remeika, in Proceedings of the Thirteenth Colloque Ampere (North-Holland Publishing Co., Amsterdam, 1964); Phys. Letters 10, 14 (1964).

${ }_{2}$ C. E. Johnson, T. E. Cranshaw, and M. S. Ridout, in Proceedings of the International Conference on Magnetism, Nottingham, 1964 (Institute of Physics and the Physical Society, London, 1965), p. 459.

${ }^{3}$ C. R. Kurkjian and D. N. E. Buchanan, Phys. Chem. Glasses 5, 63 (1964)

${ }^{4}$ H. H. Wickman, M. P. Klein, and D. A. Shirley, Phys. Rev. 152,345 (1966).
The unpaired spins of paramagnetic materials give rise to large internal fields, but these fields usually fluctuate so rapidly in direction (due to rapid electronic relaxation), that the Mössbauer effect measures only an average value, which is zero in the absence of an external field. This gives rise to a magnetically unsplit absorption line. However, in paramagnetic salts with an odd number of electrons, magnetic hfs can be observed in the absence of macroscopic magnetization if the elec-

${ }^{5}$ F. E. Obenshain, L. D. Roberts, C. F. Coleman, D. W. Forester, and J. O. Thomson, Phys. Rev. Letters 14, 365 (1965)

${ }^{6} \mathrm{G}$. Lang and W. Marshall, Proc. Phys. Soc, (London) 87, 3 (1966).

${ }^{7}$ L. Epstein (private communication).

${ }^{8}$ L. Campbell, F. deS. Barros, and S. DeBenedetti, Bull. Am. Phys. Soc. 9, 634 (1964); L. Campbell and S, DeBenedetti, Phys. Letters 20, 102 (1966). 